\title{
La interferencia lingüística de frecuencia
}

\author{
Carlos I. Echeverría Arriagada* \\ Universidad Chileno-Británica de Cultura \\ Universidad de Santiago de Chile
}

\begin{abstract}
Resumen
Este artículo trata sobre un fenómeno de contacto interlingüístico que, a pesar de haber sido reconocido en alguna de sus formas por varios estudiosos, no ha recibido aún una caracterización del todo precisa: la interferencia lingüística de frecuencia. Específicamente, se plantean los siguientes objetivos: 1) caracterizar en forma satisfactoria dicho fenómeno, 2) reflexionar en torno a su perceptibilidad y si debiera o no censurarse de modo genérico en la formación de bilingües y 3 ) hacer algunas observaciones relativas al uso de términos como anglicismo de frecuencia. Las principales conclusiones a las que se llega son: 1) la interferencia de frecuencia consiste en una desviación cuantitativa con respecto a la lengua pertinente producto del conocimiento que tiene el hablante de otra lengua, desviación que puede ser interlingüísticamente convergente o divergente; 2) no existe razón para censurar tal fenómeno de modo genérico, y, si la hubiese, habría también que censurarlo en su forma interlingüísticamente divergente; 3) los términos como anglicismo de frecuencia han de reservarse para casos interlingüísticamente
\end{abstract}

\footnotetext{
Para correspondencia, dirigirse a: Carlos I. Echeverría Arriagada (echeverria.arriagada@ gmail.com), Universidad Chileno-Británica de Cultura, C/ Santa Lucía 124, Santiago, Santiago, Chile.
} 
convergentes del fenómeno, pero nunca para referirse a los recursos lingüísticos sobreutilizados en sí mismos.

Palabras clave: lenguas en contacto, interferencia lingüística de frecuencia, educación bilingüe, términos como anglicismo de frecuencia.

\title{
LINGUISTIC INTERFERENCE OF FREQUENCY
}

\begin{abstract}
This article deals with a cross-linguistic contact phenomenon which, in spite of having been acknowledged in some of its forms by several scholars, has not yet been given a precise characterization: linguistic interference of frequency. Specifically, the following objectives are herein raised: 1) to characterize this phenomenon satisfactorily, 2) to reflect upon its perceptibility and whether it should or should not be condemned in bilingual education, and 3) to make some remarks on the use of terms such as frequency Anglicism. The main conclusions reached are the following: 1) interference of frequency consists in a quantitative deviation in relation to the relevant language due to the speaker's knowing another language, a deviation that may be cross-linguistically convergent or divergent; 2) there is no reason to condemn such a phenomenon generically, and, if there were one, one would also have to condemn it in its cross-linguistically divergent form; 3) terms such as frequency Anglicism must be used only to refer to cross-linguistically convergent cases of the phenomenon, but never to refer to the linguistic elements being overused themselves.
\end{abstract}

Keywords: languages in contact, linguistic interference of frequency, bilingual education, terms such as frequency Anglicism.

Recibido: 22/01/15 Aceptado: $15 / 03 / 16$ 


\section{INTRODUCCIÓN ${ }^{1}$}

El presente artículo, el cual nace de un afán de precisión científica y también de una preocupación didáctica, trata sobre un fenómeno de contacto interlingüístico que, a pesar de haber sido reconocido en alguna de sus formas por varios estudiosos, no ha recibido aún, a nuestro juicio, una caracterización del todo precisa: la interferencia lingüística de frecuencia. Específicamente, nos proponemos aquí: 1) caracterizar en forma satisfactoria dicho fenómeno, 2) reflexionar en torno a su perceptibilidad y si debiera o no censurarse de modo genérico en la formación de bilingües y 3 ) hacer algunas observaciones relativas al uso de términos como anglicismo de frecuencia.

Sin embargo, antes de intentar alcanzar dichos objetivos, cada uno de los cuales contará con su propia sección en el artículo (\$§ 2, 3 y 4, respectivamente), será necesario hacer algunas distinciones y precisiones de orden general en torno a las lenguas y a la interferencia lingüística. A ello dedicaremos la primera sección, en la cual se revisarán los conceptos de sistema y de norma de Coseriu (1982b) (§1.1) para luego abordar de manera muy sucinta algunos de los problemas relacionados con la delimitación de la interferencia lingüística en general y esbozar una caracterización del fenómeno (§ 1.2).

Por lo que respecta a los ejemplos proporcionados a lo largo del artículo, los idiomas en contacto considerados hipotéticamente serán el español, idioma en el que están escritas estas páginas, y el inglés, reconocido ampliamente como lengua vehicular global (vid. Mauranen 2009).

1 El presente artículo se basa principalmente en el marco conceptual de Echeverría 2015, investigación que contó con el financiamiento parcial de una Beca de Incentivo a la Investigación otorgada por el Vicedecanato de Investigación y Postgrado de la Facultad de Humanidades de la Universidad de Santiago de Chile. Corresponden a versiones parciales de este artículo, aquí refundidas, adaptadas y ampliadas, las ponencias Echeverría 2014a y 2014b. 


\section{DISTINCIONES PREVIAS}

\subsection{SisTEMA Y NORMA DE LA LENGUA}

En el que quizá sea su más conocido ensayo, Coseriu (1982b), desarrollando un planteamiento de Hjelmslev y Lotz (ápud Coseriu 1982b: 11), señala las deficiencias de la dicotomía saussureana "lengua-habla" (Saussure 2005) $\mathrm{y}$, tomando en todo momento como punto de partida el hablar concreto, "única realidad investigable del lenguaje" (1982b: 94), propone sustituir dicha dicotomía con una distinción tripartita en la realidad del lenguaje. De ahí el nombre del ensayo: Sistema, norma y habla.

Para el maestro rumano, la lengua, como entidad susceptible de una descripción sistemática, se constituye como una abstracción en que se recogen los aspectos comunes comprobados en los actos lingüísticos de una comunidad, o sea, como un sistema de isoglosas en el sentido amplio (no puramente geográfico) del término (vid. Pisani 1947). Al concebir así la lengua, llega Coseriu a la conclusión de que esta no puede reducirse a un sistema de oposiciones indispensables para la comunicación, es decir, a un sistema de invariantes funcionales, como p. ej., los fonemas, según se propone en el Curso de Saussure, sino que debe entenderse también como un conjunto de usos lingüísticos repetidos, normales:

No sólo las invariantes, sino también las variantes normales, se dan en número limitado en cada lengua y caracterizan la lengua misma. Es decir que existen en cada lengua oposiciones constantes y peculiares, tanto entre las invariantes como entre las variantes normales, con la diferencia de que las oposiciones entre invariantes son funcionales, mientras que las oposiciones entre variantes no tienen tal carácter, aun no siendo ni indiferentes ni arbitrarias en la lengua dada. $\mathrm{O}$ sea que existen aspectos extrafonológicos y, en general, extraestructurales, adicionales, no pertenecientes al sistema y que, sin embargo, no se dan como puramente casuales, sino que caracterizan una lengua... (1982b: 68).

De este modo, según este lingüista, en la descripción de las lenguas han de distinguirse dos niveles sucesivos de formalización: el del sistema normal, o norma, que contiene "sólo lo que en el hablar concreto es repetición de modelos anteriores" (1982b: 95), y el del sistema funcional, o simplemente sistema, que contiene "sólo lo que en la norma es forma indispensable, oposición funcional, habiéndose eliminado [...] todo lo que en la norma es simple costumbre, simple tradición constante [...] sin valor funcional" (1982b: 96). 
Así, p. ej., en español en general, a diferencia de lo que sucede en lenguas como el francés, no existe a nivel de sistema oposición entre vocales abiertas y cerradas; no importa, pues, en términos funcionales, si /e/ se realiza como abierto o como cerrado, pues se tratará siempre del mismo fonema. Sin embargo, a nivel de norma se constata que en ciertos contextos fónicos dicho fonema se realiza como cerrado (p. ej., en queso, cabeza, sello, etc.), mientras que en otros se realiza como abierto (p. ej., en papel, afecto, peine, etc.). Y, pasando del ámbito fónico al morfológico, en inglés en general, a nivel de sistema oxes ("bueyes") corresponde a una formación del todo válida, ya que en dicha lengua $o x$ significa "buey" y la terminación -es funciona, en efecto, como pluralizadora (p. ej., en mazes ["asnos"], aces ["ases"], places ["lugares"], etc.). Sin embargo, a nivel de norma, oxes se constata solo de manera muy excepcional como realización efectiva, siendo oxen la opción normal, independientemente de que, desde el punto de vista del contenido ${ }^{2}$, ambas formas sean funcionalmente intercambiables.

\subsection{LA INTERFERENCIA LINGÜÍSTICA}

Aunque ya en 1938, en el IV Congreso Internacional de Lingüistas, Sanfeld y Jakobson (ápud Blas 1991: 266) aludían al concepto de interferencia lingüística en sus contribuciones, comúnmente $-\mathrm{y}$ no sin motivos- se cita como responsable de la incorporación definitiva de dicho concepto en la ciencia del lenguaje a Weinreich, quien escribió:

Los casos de desviación con respecto a las normas de cualquiera de las dos lenguas que ocurren en el habla de los individuos bilingües como resultado de su familiaridad con más de una lengua, es decir, como resultado de contactos, serán denominados fenómenos de interferencia (1974: 17).

Desde la publicación de la obra de este lingüista, la cual ha tenido un impacto innegable en el estudio del contacto entre lenguas, bastante ha sido lo que se

2 Usaremos los términos expresión y contenido, según la tradición glosemática (vid. Hjelmslev 1971), para referirnos, sin distinguir todavía entre forma y sustancia, a los dos planos del lenguaje: al plano del significante y al del significado, respectivamente, según la terminología saussureana (Saussure 2005). 
ha hablado sobre el tema en artículos, comunicaciones y libros; sin embargo, en realidad no ha sido mucho el consenso que ha habido con respecto a qué ha de entenderse exactamente por interferencia, cosa que pone de manifiesto, entre otros, Blas (1991), quien señala los problemas que supone la delimitación de la interferencia en relación con el "error" lingüístico, el bilingüismo y el cambio lingüístico ${ }^{3}$. Por lo anterior, si bien no es nuestro propósito en esta ocasión caracterizar de modo exhaustivo la interferencia lingüística en general, resulta crucial aclarar algunos puntos al respecto.

Aquí, partiendo de la clásica definición de Weinreich, pero añadiendo algunas precisiones, provisionalmente definiremos la interferencia lingüística como una desviación, en el habla de sujetos bilingües ${ }^{4}$, producto de su condición de tales, con respecto a la norma o al sistema de la lengua pertinente, a saber, la lengua que se toma como modelo para la consideración de la producción lingüística del hablante por las circunstancias (reales o imaginarias $^{5}$ ) del acto comunicativo y que corresponde a una de las lenguas conocidas (al menos de modo parcial) por esos sujetos ${ }^{6}$. Por tanto, acerca de los temas en relación con los que Blas señala la existencia de problemas de delimitación de la interferencia, puede afirmarse lo siguiente: 1 ) en cuanto a si los fenómenos de interferencia implican errores (o equivocaciones, si es que se hace la distinción [vid. Corder 1967, Brown 2000]), todo dependerá de qué se entienda por error (y equivocación): si se entiende una desviación cualquiera con respecto a la lengua propia de la comunidad y de las circunstancias comunicativas en que se inserta o se pretende insertar discursivamente el hablante -así como con respecto al saber lingüístico correspondiente-, la respuesta será positiva; en cambio, si se entiende una desviación que necesariamente se da también con respecto al sistema de esa lengua -que es la forma en que nosotros nos atreveríamos a usar el término-, la respuesta solo será positiva para algunos casos, ya que también hay interferencias solo

3 Blas trata por separado los problemas de delimitación de la interferencia en relación con el cambio lingüístico y con la integración, pero en verdad se trata de un mismo fenómeno (vid. Coseriu 1978, Mackey 1970).

4 Es decir, sujetos que cumplan con la condición de hablar al menos dos lenguas, independientemente de que hablen más.

5 Pueden ser circunstancias imaginarias en el aula de lengua extranjera, donde es común hacer a los estudiantes imaginar que se están comunicando con hablantes nativos.

6 El uso de términos como lengua meta al hablar de interferencia nos parece problemático, ya que determinar qué lengua ideal busca realizar el hablante, o si tan siquiera busca realizar una lengua en particular, es bastante difícil, especialmente por lo que concierne a cuestiones de pura norma. Por esto, aquí preferimos usar el término lengua pertinente, considerando el contexto idiomático (vid. Coseriu 1982a) del acto comunicativo. 
de norma; 2) solo podrá haber interferencia propiamente dicha en el habla de quienes conozcan, al menos de modo parcial, la lengua pertinente y la lengua desde la que se produce el fenómeno; las desviaciones de hablantes que no cumplan con esa condición que se hayan originado por una situación de bilingüismo social solo serán indirectamente interferenciales; 3) toda interferencia propiamente dicha supondrá una innovación con respecto a la lengua pertinente, es decir, una desviación sincrónica; en consecuencia, los usos que se hayan originado como interferencias pero que luego hayan sido adoptados por la masa también serán, desde su difusión, solo indirectamente interferenciales, y esta vez solo al ser considerados desde una perspectiva diacrónica. Por otra parte, se desprende de la definición proporcionada que nosotros consideraremos la interferencia en cuanto fenómeno de habla, es decir, en cuanto fenómeno del lenguaje realizado, aunque es evidente que tiene también un aspecto psicolingüístico, al cual por razones obvias la lingüística aplicada al aprendizaje de lenguas extranjeras ha prestado particular atención (vid. Barkman 1968; Brown 2000; Ellis 1994; Gass, Behney y Plonsky 2013; Lado 1973; McLaughlin 1984; Odlin 1989).

Así, podrá hablarse de interferencia, p. ej., frente al uso de actually con el contenido "actualmente" por parte de un hispanohablante aprendiente de inglés al intentar comunicarse con un hablante de esta última lengua, en la que actually significa "realmente" (interferencia de sistema); frente al uso de esp. controversial por parte de un angloparlante aprendiente de español al comunicarse con un hispanohablante, por imitación del uso de la palabra homógrafa del inglés, poseedora de un significado análogo, en tiempos en que aquella, a pesar de ser una formación posible según el sistema hispánico, aún no se constataba como realización efectiva, siendo lo común usar controvertido (interferencia solo de norma) ${ }^{7}$; o frente al uso de déjame solo, por parte del mismo anglohablante y en la misma situación anterior, para solicitar a un destinatario abusivo que deje de molestarlo, por imitación del uso análogo que se suele dar a ingl. leave me alone ("déjame solo") (también interferencia solo de norma).

\footnotetext{
En la Hispanoamérica de la actualidad, la misma situación no implicaría interferencia, pues controversial es ya una palabra bastante común en dicho territorio, e incluso es reconocida por la Real Academia Española (2014).
} 


\section{CARACTERIZACIÓN DE LA INTERFERENCIA LINGÜÍSTICA DE FRECUENCIA}

Desde que comenzó a hablarse de interferencia en la ciencia del lenguaje, gran parte de los estudiosos que han tratado el tema -varios de los cuales han publicado obras que podrían considerarse clásicas en el estudio del contacto entre lenguas y en la lingüística aplicada a la enseñanza y al aprendizaje de lenguas extranjeras- parecen haber concebido la interferencia lingüística como una desviación consistente en el uso o el almacenamiento en el acervo lingüístico de uno o más elementos (simples o complejos) que, como unidades delimitables, constituyen en sí mismos anomalías (innovaciones) respecto de la lengua que se toma como modelo (vid. Baetens 1986; Barkman 1968; Brown 2000; Debyser 1970; Dubois et al. 2002; Gass, Behney y Plonsky 2013; Haugen 1956; Hernández 1998; Lado 1973; López 1993; Mackey 1970; McLaughlin 1984; Odlin 1989; Overbecke 1976; Thomason 2001; Thomason y Kaufman 1988; Weinreich 1974). En otras palabras, la interferencia lingüística, así entendida, consistiría siempre en una desviación cualitativa, sea solo respecto de la norma de la lengua o también respecto de su sistema, como sucede con los ejemplos proporcionados en $\S 1.2$.

Sin embargo, ha habido estudiosos que han ampliado el concepto de interferencia lingüística, haciéndolo extensivo también a desviaciones ya no cualitativas, sino cuantitativas, que son las que designaremos con el término interferencia de frecuencia (vid. Alcaraz y Martínez 1997; Coseriu 1977; Kabatek 1997a, 1997b, 2000; Granda 1968; Vázquez-Ayora 1977) ${ }^{8}$. En lo que sigue, con el fin de llegar a una caracterización satisfactoria del fenómeno, nos referiremos en relativo detalle al planteamiento desarrollado por dos de estos estudiosos, que son quienes, a nuestro parecer, han abordado en forma más esclarecedora, desde el punto de vista teórico, el fenómeno de la interferencia lingüística: Coseriu (1977), de cuya visión tripartita del lenguaje hemos hablado antes, y su discípulo Kabatek (1997a, 1997b, 2000). Citaremos aquí casi exclusivamente el artículo de Kabatek titulado Dime cómo hablas y te diré quién eres (1997a), que ofrece la ventaja de estar escrito en español, y nos referiremos al trabajo de Coseriu solo para aclarar aquello que en la formulación de su discípulo no quede del todo precisado.

8 Gómez (1998), p. ej., también reconoce tales desviaciones, pero él no habla de interferencias, sino de préstamos de frecuencia. 
Kabatek, siguiendo a su maestro, distingue dos tipos fundamentales de interferencia: la positiva, que produce "resultados directamente comprobables en los discursos producidos", consistentes en "elementos positivamente presentes", y la negativa, que consiste en "la no-realización de ciertos elementos" (1997a: 223). Dentro de la interferencia positiva, distingue este autor la interferencia de transposición, que "consiste en transponer elementos del sistema B a un discurso del sistema A" (1997a: 222), y la hipercorrección, "que resulta de la aplicación de unas reglas de conversión de elementos de $\mathrm{B}$ en elementos de A también en casos donde los elementos forman parte, en realidad, de la zona convergente AB" (1997a: 223). Y dentro de la interferencia negativa, distingue la interferencia de convergencia, que "corresponde a la realización preferente de los elementos de la zona de confluencia $\mathrm{AB}$, tanto por razones de economía lingüística como también por desconocimiento de los elementos divergentes" (1997a: 223), con la consecuente "realización reducida o 'realización negativa' de elementos de la zona divergente (por ejemplo, en un texto de A, de la zona $\mathrm{A}$ $\sin A B)$ " (1997a: 223-224), y la interferencia de divergencia, que corresponde a "la realización proliferada de los elementos divergentes (en un texto de A, de la zona $\mathrm{A} \sin \mathrm{AB}$ )", con la "consecuente realización menos frecuente o 'negativa' de los elementos de la zona común" (1997a: 223).

Recurriendo a los conceptos coserianos de sistema y norma, Kabatek explica lo siguiente sobre los dos tipos fundamentales de interferencia por él identificados:

En el caso de las interferencias positivas, hay que distinguir entre interferencias que afectan el sistema de una lengua e interferencias que no afectan el sistema pero que producen alteraciones de la norma. Las interferencias negativas, en cambio, sólo pueden producir alteraciones de la norma de una lengua, alterando solamente la frecuencia normal de realización de ciertos elementos (1997a: 224).

De estas dos clases interferenciales, en esta ocasión naturalmente nos centraremos en la llamada interferencia negativa, que es en la que se agrupan los fenómenos consistentes en desviaciones cuantitativas.

Como se podrá advertir, en esta clase entran no solo las desviaciones consistentes en el sobreuso de elementos de la lengua pertinente que encuentran un elemento correspondiente en la lengua desde la que se produce la interferencia (casos de interferencia de convergencia; $p$. ej., en el caso de un hispanohablante con conocimientos de inglés, el sobreuso de adverbios en -mente -suponiendo que exista en español o en la variedad considerada una cierta regularidad en cuanto a la frecuencia de uso de dichos adverbios- por imitación del hábito anglosajón de usar en abundancia adverbios en -ly), 
sino también las desviaciones consistentes en el infrauso de tales elementos (casos de interferencia de divergencia; $p$. ej., en el caso de alguien que realiza una traducción del inglés al español, el infrauso del gerundio -nuevamente, suponiendo que exista una cierta regularidad en cuanto a la frecuencia de uso del gerundio-, por temor a emplearlo de manera errónea por imitación del uso de las formas anglosajonas en -ing halladas en el texto original). Esto constituye uno de los motivos por los que el planteamiento desarrollado por Coseriu y Kabatek resulta superior a otros. En efecto, la mayoría de los estudiosos que han considerado la posibilidad de hablar de interferencias de tipo cuantitativo parece considerar solo el primer tipo de desviación (vid. Alcaraz y Martínez 1997; Gómez 1998; Granda 1968; Lorenzo 1980, 1996; Vázquez-Ayora 1977); pero Coseriu y Kabatek logran darse cuenta de que, en rigor, frente a tales casos, aquellos en que se constata que la frecuencia con que se usa un determinado recurso es menor que la normal, en cuanto fenómenos de interferencia, no tendrían por qué recibir un tratamiento diferente, al menos desde un punto de vista resultativo?.

Pues bien: a pesar de que Kabatek y Coseriu identifican con bastante claridad los dos tipos de fenómenos interferenciales de tipo cuantitativo, existen en el planteamiento desarrollado por estos autores al menos dos problemas.

El primer problema, el cual surge en la exposición de Kabatek, es que este lingüista, al hablar de una zona de confluencia de las lenguas que conoce el hablante que incurre en la llamada interferencia negativa, entendida como una zona interlingüística abstracta cuyos elementos el hablante preferirá o evitará (según se trate de un caso de interferencia de convergencia o de divergencia, respectivamente), parece dar por sentado que siempre habrá identidad entre los recursos de la lengua pertinente que son sobre- o infrausados y los recursos correspondientes de la otra lengua a causa de los que se ocasiona la interferencia, lo cual constituiría un error. Esto, en cambio, no sucede en la exposición de Coseriu (1977), quien habla de recursos idénticos o análogos.

El segundo problema, el cual requerirá mayor disquisición, se halla tanto en el trabajo de Coseriu como en el de Kabatek, y es que la clase más general a la que la interferencia de convergencia y la de divergencia pertenecen no es caracterizada de manera adecuada. Como ya se ha mencionado, para dichos estudiosos la interferencia de convergencia y la de divergencia

9 L. Payrató (ápud Kabatek 1997a: 224, n. 27) reconoce también la interferencia de divergencia, aunque él habla de ultracorrección. 
pertenecen a la clase de la interferencia negativa, por cuanto en ambos casos la desviación estaría dada por la no-realización, o realización negativa, de ciertos elementos lingüísticos. No obstante, la "negatividad" constatada en estos tipos interferenciales no es realmente una nota distintiva de ellos, ya que también puede observarse en otros fenómenos de interferencia. Así, p. ej., si un hablante nativo de inglés que está aprendiendo español, al intentar hablar esta lengua, emplea ingl. army ("ejército"), no solo tendremos la realización de dicha palabra, sino también la consecuente no-realización de ejército, así como de otras alternativas frásticas ("explicativas") que ofrece la lengua española; y, de modo análogo, si el mismo hablante, en las mismas circunstancias, emplea indoctrinación por imitación de ingl. indoctrination, tendremos la no-realización de adoctrinamiento y de otras alternativas hispánicas. Todo caso de interferencia $-\mathrm{y}$, en realidad, todo acto lingüísticotendrá, pues, dos caras: una positiva, consistente en la realización de ciertos elementos, y una negativa, consistente en la consecuente no-realización de otros. Por tanto, establecer una clase de interferencia negativa, como opuesta a la de la interferencia positiva, no es una solución satisfactoria.

Lo que realmente distingue a la interferencia de convergencia y a la de divergencia no es su "negatividad", sino ni más ni menos que su índole de desviación cuantitativa, dada solo por la frecuencia de uso de un recurso lingüístico que por lo demás es castizo. Es por esto por lo que aquí, para referirnos a la clase a que pertenecen ambos fenómenos, optamos por emplear el término interferencia de frecuencia, que tomamos de Alcaraz y Martínez (1997) -quienes no obstante definen el fenómeno designado de manera bastante deficitaria ${ }^{10}$-. Concretamente, por interferencia de frecuencia se

10 En palabras de Alcaraz y Martínez: "La interferencia de frecuencia consiste en usar elementos que sí existen en la lengua meta, pero que no se utilizan con tanta regularidad como en la lengua original (por ejemplo, la pasiva en español y en inglés)" (1997: 303-304). El problema más grave (pero lamentablemente no el único) de esta definición es que, de tomársela al pie de la letra, se terminarían por considerar como interferenciales usos que no implican desviación alguna con respecto a la lengua pertinente (o "lengua meta"); así, de acuerdo con las palabras de Alcaraz y Martínez, si se acepta que las construcciones pasivas con ser + participio del español son menos frecuentes que las construcciones correspondientes del inglés, habría que aceptar que se producirá interferencia con la sola aparición de aquellas en el habla de un hispanohablante con conocimientos de inglés, sin importar la frecuencia constatada en dicho uso, lo cual resulta un sinsentido, porque se trata de un recurso totalmente castizo en español, proveniente del latín. Parece bastante claro, pues, que estos autores no consideran como fenómeno de interferencia de frecuencia el mero empleo de construcciones pasivas con $s e r+$ participio (ni el de ningún otro recurso propio de la "lengua meta"), sino solo su uso con una frecuencia mayor que la normal constatada, por lo cual habría que reemplazar la palabra usar por sobreusar en la definición citada. A este respecto, vid. § 4 . 
entenderá aquí el empleo de un recurso constatado en la norma de la lengua pertinente $(y$, por tanto, válido según el sistema correspondiente) con una frecuencia atípica respecto de la registrada para ese recurso en dicha norma, producto del conocimiento que tiene el sujeto hablante de otra lengua.

Definida de este modo, la interferencia de frecuencia ha de distinguirse de otros fenómenos que, si bien en primera instancia podrían confundirse fácilmente con ella, son en realidad de diferente índole. Así, no hay interferencia de frecuencia cuando se presenta el uso reiterado, por motivo de interferencia, del significante de un recurso de la lengua pertinente con un contenido impropio según el sistema, fenómeno en que en rigor lo que lo que se tiene, si se considera tanto expresión como contenido, no es el empleo de un recurso de la lengua pertinente, sino de un recurso nuevo ( $p$. ej., el uso de formas gerundioides con valor nominal al traducir del inglés al español, por imitación de los usos dados a las formas terminadas en -ing en el texto original). Por otro lado, cuando se presenta el uso reiterado de recursos de la lengua pertinente con funciones de habla anormales (pero no imposibles en términos funcionales) positiva o negativamente (p. ej., en una traducción del inglés al español, el uso no enfático del pronombre personal en un contexto en que la forma normal de usar el pronombre sería con función enfática), o con la consecuencia de extrañeza o molestia en el destinatario (p. ej., el uso, en una traducción del inglés al español, de adverbios en -mente muy cerca unos de otros y por tanto con efecto cacofónico), en caso de haber interferencia de frecuencia, habrá también algo más, que no será consecuencia necesaria de esta ${ }^{11}$.

Ahora bien: ya definido y en gran parte deslindado el fenómeno, es de vital importancia aclarar que, en rigor, solo se puede hablar de interferencia de frecuencia si existe una cierta regularidad en la lengua pertinente en cuanto a la frecuencia de empleo del recurso considerado. Kabatek, p. ej., parece confundido a este respecto cuando, al referirse a las dificultades inherentes al descubrimiento de los fenómenos de interferencia de frecuencia, afirma que "resulta casi imposible definir lo que es la frecuencia 'normal' de elementos en un texto, sobre todo si es un texto producido en una situación de conflicto lingüístico con normas poco estables" (1997a: 224, n. 28). El problema está en que la interferencia de frecuencia (y la interferencia en general) es siempre un hecho de habla que se determina en relación con un hecho de lengua, y todo hecho de lengua es, como ya hemos dicho (§ 1.1), un hecho

11 De hecho, al presentarse un fenómeno de interferencia de frecuencia junto con el empleo reiterado del recurso correspondiente de la lengua pertinente con una función de habla anormal, este último fenómeno, de interferencia cualitativa, será racionalmente anterior. 
de isoglosas, es decir, un hecho de regularidad de uso. Por tanto, si no existe estabilidad alguna en cuanto a la frecuencia de empleo de un recurso en el conjunto de actos lingüísticos de la comunidad considerada, simplemente no habrá un hecho de lengua con que se pueda hacer la comparación que lleve a determinar si se está o no ante un caso de interferencia de frecuencia. En otras palabras, no puede hablarse de anormalidad si no existe una normalidad previa identificable12.

\section{SOBRE LA PERCEPTIBILIDAD Y LA CENSURA DE LA INTERFERENCIA LINGÜÍSTICA DE FRECUENCIA}

Es bien sabido que el término interferencia en el ámbito del estudio del lenguaje tiene una connotación más bien negativa. Como escribe Blas: "[E]l término interferencia nació bajo la óptica de un valor negativo, de su consideración de 'ataque' a las normas del sistema. En este sentido, hablar de interferencia era hablar de "error"' (1991: 267). De este modo, no es infrecuente que, al hablarse de fenómenos de interferencia, sobre todo en el ámbito de la enseñanza de la traducción y de lenguas extranjeras, ello sea con un propósito censurador, es decir, para advertir a los hablantes sobre lo nocivo que resulta el fenómeno y proporcionarles herramientas para que no incurran en él. Y esto también se extiende al tratamiento de la interferencia de frecuencia, aunque al parecer solo en su forma de interferencia de convergencia. Un ejemplo es el de Vázquez-Ayora, quien a propósito de los anglicismos de frecuencia - de los que se hablará en la próxima sección-en la traducción del inglés al español escribe:

Los anglicismos sintácticos, queremos recalcar, son tan dañinos como los léxicos [...]. Hay casos en que la forma sintáctica o el vocablo son correctos, pero es la 'frecuencia' la que perturba el canal de comunicación

12 Es de todas maneras importante tener en cuenta que la regularidad y la normalidad, como condiciones absolutas, son condiciones ideales, tal como la lengua estática es un objeto ideal, deducido por el lingüista mediante un procedimiento abstractivo (vid. § 1.1 y Coseriu 1978: 50-51). De este modo, podremos preguntarnos según qué convención estadística determinaremos en cada caso el límite entre, por un lado, lo regular y lo irregular, y, por otro lado, lo normal y lo anormal; pero tal pregunta tendrá un carácter más bien metodológico que teórico. 
con elementos ajenos al espíritu del español, que se imponen sobre los que le son propios, y dan a la versión un sabor extraño y la falta de autenticidad. El estudio de los 'anglicismos de frecuencia' tiene pues trascendencia lingüística, sobre todo en nuestra disciplina, y los métodos de evitarlos deben formar parte del inventario de técnicas de la traductología (1977: 102-103, cursivas nuestras).

Cabe en este punto preguntarse: ¿en qué medida se justifica esta actitud purista? Para intentar dar respuesta a este interrogante, nos permitiremos una breve digresión, en la que nos referiremos a las observaciones que hace Coseriu en relación con el tema de los americanismos hispánicos:

sacaclavos es "americanismo" sólo porque en este sentido la norma española ya tiene consagrado el término desclavador. Y papal, en el sentido de "plantación de papas" no es "americanismo" sino desde el punto de vista de la norma actual de España, mientras que desde el punto de vista del sistema es formación de lo más castiza. En efecto, palabras perfectamente españolas se crean no sólo en España, sino también en América, porque también en América funciona el sistema lingüístico español y, si las palabras nuevas representan realizaciones de posibilidades del sistema, nada importa que hayan surgido en Madrid o en Montevideo (1982b: 79).

En el fragmento citado - que es parte de una argumentación mayor sobre la validez en general de las creaciones lingüísticas que surgen de las posibilidades funcionales de la lengua-, al defender el carácter propiamente castizo de las creaciones lingüísticas americanas nacidas según el sistema panhispánico, Coseriu evidentemente da más importancia al sistema que a la norma en lo relativo a la valoración de las innovaciones de los hablantes; y esto incluso si las innovaciones no vienen a llenar "vacíos" expresivos del idioma, sino que más bien surgen como alternativas a formas preexistentes, como en el caso de sacaclavos frente a desclavador. En efecto, desde el punto de vista comunicativo, si un individuo no habla según la norma constituida, pero de todos modos realiza de manera correcta las posibilidades del sistema, siempre se tratará de un uso menos reprochable que otro en el que esto no ocurra, incluso si las anomalías lingüísticas causan extrañeza y aun dificultad de comprensión, pues mientras exista un sistema común existirá la posibilidad racional de comunicación. Y si con sus innovaciones el hablante no solo realiza las posibilidades del sistema, sino que además no da lugar a ningún efecto indeseado en el destinatario, la verdad es que ya nada podrá reprochársele desde este punto de vista; será, en términos comunicativos, un uso tan lícito como uno basado por completo en la norma constituida. 
En el caso de la interferencia de frecuencia, ya se ha dicho que esta, en cuanto fenómeno interferencial, se caracteriza solo por su naturaleza de desviación cuantitativa, y que si bien puede presentarse junto con otros fenómenos entorpecedores de la comunicación, estos no son una consecuencia necesaria de aquella. Por ello, no es extraño que haya casos de interferencia de frecuencia adscritos al último tipo de innovación mencionado ${ }^{13}$, a saber, al que no implica problema comunicativo alguno. Más aún: por la naturaleza del fenómeno, que hace que la desviación se manifieste no en unidades discretas del decurso lingüístico, sino a lo largo de todo este -"sin localizarse en ninguna parte", diría Vázquez-Ayora (1977: 202)-, no pocas veces ocurrirá que, frente a casos de interferencia de frecuencia, el destinatario, aun si es el más leído, no solo no sentirá extrañeza, sino que ni siquiera en un intento reflexivo será capaz de darse cuenta de que está ante una anomalía. En efecto, rara vez las personas están pendientes de la frecuencia con que se emplea tal o cual recurso lingüístico al asumir el rol de destinatarios, y si el docente de lengua extranjera o de traducción es capaz de detectar a simple vista la anomalía en cada caso - cosa que es improbable a menos que se haga un análisis detenido de las producciones de los alumnos, las cuales, además, la mayoría de las veces serán muy breves como para sacar conclusiones estadísticas significativas-será por deformación profesional. A este respecto, puede resultar esclarecedor citar a Viaggio, quien, a propósito del concepto de marcación, escribe:

Me apresuro a aclarar, una vez más, que la marca formal [...] solo funciona si se capta. No hay más forma que la percibida: en la pintura solo cuentan los colores del espectro visible y en la música lo que el oído humano puede distinguir. Si la cantidad de eñes de este libro es exactamente el cuadrado de las rimas en -ava del "primer canto" de la Divina Comedia, al margen de que haya tenido o no la intención consciente de que así sea, no hay manera de percibirlo. Ergo, no cuenta (2004: 320).

13 Algunos autores probablemente rechazarían la idea de que los fenómenos de interferencia de frecuencia constituyen innovaciones lingüísticas. P. ej., Pountain (1994), al tratar el tema del anglicismo sintáctico hispánico, distingue entre fenómenos de innovación y de explotación, refiriéndose a anglicismos de sistema y de norma, respectivamente (aunque sin usar la terminología coseriana). Sin embargo, la verdad es que todo fenómeno de interferencia lingüística, sea de sistema o de norma, implicará una innovación en el sentido amplio del término (vid. Coseriu 1978). En el caso de la interferencia de frecuencia, la innovación será, como se ha dicho, cuantitativa. 
Así pues, si hay, en una situación de contacto entre el inglés y el español, o en cualquier situación comunicativa hispánica, p. ej., un aumento en el uso de adverbios en -mente con respecto a lo normalmente constatado en español, ello nada importará si el destinatario ni siquiera es capaz de notar que hay algo atípico acerca de dicho uso, que es muy probablemente lo que sucederá mientras el emisor procure no abandonar por completo otras construcciones normales del español y no utilizar los adverbios muy cerca unos de otros, lo que causaría cacofonías. Habrá, sí, una anomalía real, constatable mediante un análisis estadístico ulterior; pero no será una realidad perceptible para el destinatario en la inmediatez del discurso.

Por lo hasta ahora expuesto, resulta sensato afirmar que no hay una verdadera justificación para censurar de manera genérica la interferencia de frecuencia, rehuyéndola como si se tratase de algo intrínsecamente nocivo. Tal actitud solo es reflejo de un dogmático purismo, por completo infértil para cualquier propósito de planificación lingüística, ya que olvida que la lengua no es algo que se imponga al individuo sin más, sino que, en cuanto sistema, "se le ofrece, proporcionándole los medios para su expresión inédita, pero, al mismo tiempo, comprensible, para los que utilizan el mismo sistema" (Coseriu 1982b: 982) ${ }^{14}$.

Desde luego, un buen docente de lengua extranjera o de traducción debe conocer bien los fenómenos como la interferencia de frecuencia, así como debe también sensibilizar al estudiante respecto de tales fenómenos y familiarizarlo con los problemas (por cierto condenables) con que estos se relacionan: ello forma parte de la misión que tiene el docente de concienciar en forma constante al alumno en relación con su actividad. Mas no resulta pertinente censurar de manera tajante un fenómeno lingüístico que, a pesar de constituir una desviación con respecto a la conducta "esperable" de un hablante en circunstancias "normales", corresponde sencillamente a una explotación particular del sistema de la lengua (vid. Pountain 1994), sin la consecuencia necesaria de problemas comunicativos y sin siquiera manifestarse necesariamente como algo perceptible para los destinatarios. Y si fuese pertinente tal censura -reiteramos: no lo es-, esta debiera implementarse de manera consecuente; o sea, no solo habría que condenar la interferencia de frecuencia en su forma convergente, sino también en su forma divergente, cosa que nadie parece hacer.

14 Aunque Coseriu en un principio hablaba de "imposiciones sociales y culturales" (1982b: 98) al referirse a la lengua en cuanto norma, finalmente admite que "era una expresión poco feliz, pues la lengua no se 'impone' a los hablantes" (1978: 56, n. 65). 


\section{SOBRE EL USO DE TÉRMINOS COMO ANGLICISMO DE FRECUENCIA}

No es poco común en la literatura especializada que, para designar, al menos en principio, casos de interferencia de convergencia producidos desde un idioma en particular, se empleen denominaciones como esp. catalanismo de frecuencia, fr. anglicisme de fréquence, ingl. frequency Gallicism, etc. (vid. Cardinal 2009; Gold 1983; Darbelnet 1976; Lorenzo 1980, 1996; Martínez 2008; Rodríguez 2000, 2002; Vázquez-Ayora 1977), en las que las palabras catalanismo, anglicisme, Gallicism, etc., son las encargadas de especificar el origen de la interferencia (en el primer caso, el catalán; en el segundo, el inglés; en el tercero, el francés). Así, p. ej., en el ámbito hispánico, Lorenzo, utiliza el término anglicismo de frecuencia -acuñado por él en 1956 (Lorenzo 1980)- para referirse al "uso inmoderado [por influencia del inglés] de ciertas palabras o expresiones que si no son anglicismos en sí, al excluir otras opciones que ofrece la lengua española, suenan, por su insistencia, extraños y monótonos" (1996: 616). Y, en la misma línea, en un clásico tratado sobre traducción, Vázquez-Ayora, quien es probablemente el que más responsabilidad tuvo en la difusión del término, escribe al respecto:

Como su nombre lo indica, [el anglicismo de frecuencia] es causado por la 'frecuencia' insólita con la que aparece algún 'giro' o 'término' sin que ese giro o término sea necesariamente un anglicismo en sí. Puede no ser giro extranjero, mas su repetición en el uso no es castiza, y en ello se distingue de las otras clases de anglicismos que hemos conocido antes (1977: 103).

Ahora bien: antes se ha dicho que la mayor parte de los estudiosos que se han referido a la posibilidad de interferencias cuantitativas solo considera casos de convergencia; no obstante, independientemente de si quienes emplean denominaciones como las que nos ocupan en este apartado están o no conscientes de que existe la interferencia de divergencia, el que dichas denominaciones se usen solo para designar casos de interferencia de convergencia está por completo justificado y ha de mantenerse así, aun si se reconocen ambas clases interferenciales. Los términos anglicismo, catalanismo, galicismo, etc., como términos metalingüísticos ${ }^{15}$, siempre

15 Hacemos la especificación ya que estos términos también podrían usarse para referirse a la imitación de conductas extranjeras en general, sean o no lingüísticas. Así, se podría decir 
designan fenómenos en que se imita otro idioma, cosa que no sucede en el caso de la interferencia de divergencia, en referencia a la cual sería más adecuado hablar de antianglicismos, anticatalanismos, antigalicismos, etc., por cuanto se trata siempre de usos que van en contra de lo observado en el idioma desde el cual se produce la interferencia.

Por otra parte, en el uso de términos como los mentados pueden en ocasiones darse diversas imprecisiones y confusiones. Sin embargo, varias de ellas no presentan en realidad mayor dificultad a la luz de lo expuesto en el último párrafo del apartado anterior, por lo que en lo que sigue nos detendremos en un problema en particular, que se puede constatar en varios trabajos en español sobre los anglicismos de frecuencia, aunque es probable que también exista en otros idiomas y en referencia a fenómenos de interferencia de convergencia de diferente origen.

Este problema se presenta, p. ej., en el libro de Vázquez-Ayora, donde se lee que "[e]ntre los 'anglicismos de frecuencia' correspondientes al léxico, cunde en primera línea el adverbio, de modo especial el terminado en -mente" (1977: 116); en el estudio de Rodríguez, donde se lee que "son anglicismos de frecuencia sintácticos los adjetivos antepuestos al sustantivo, los adverbios en -mente, el gerundio, las repeticiones innecesarias y el verbo poder" (2000: 309; 2002: 166) y donde se habla continuamente de anglicismos de frecuencia para hacer referencia a cada una de las apariciones independientes de los recursos lingüísticos hispánicos cuyo sobreuso supone un anglicismo en el corpus estudiado; y, ya desde el título, en la ponencia de Véliz y Cámara (2010): La "parataxis" como anglicismo de frecuencia en traducciones del inglés al castellano.

Lo que tienen en común de problemáticos los casos citados es que en ellos no se distingue entre el sobreuso de un recurso lingüístico dado y el recurso en sí mismo, aunque evidentemente se trata de fenómenos distintos, tanto como lo son un cardumen y los peces que lo conforman (considerados como individuos o como una clase en abstracto). En efecto, es obvio que en el caso de un cardumen los peces no adquieren en sí mismos la condición de cardúmenes por el hecho de conformar uno conjuntamente; la condición de cardumen solo está en el grupo. Algo análogo, pues, puede decirse de los fenómenos de interferencia de frecuencia. Así, p. ej., en el caso del contacto entre el inglés y el español, los adjetivos prenominales, los adverbios en -mente, las construcciones paratácticas, etc., en cuanto recursos del español,

que imitar el hábito de los ingleses de tomar té en la tarde es también un anglicismo, aunque claramente no uno lingüístico. 
sean considerados de manera concreta o abstracta, no se convierten en sí mismos en anglicismos ni se comportan como tales por el hecho de ser sobreutilizados con el resultado de anglicismos de frecuencia. En tales casos, el anglicismo no se halla en los recursos lingüísticos sobreutilizados en sí mismos, que son racional y cronológicamente anteriores, sino en los conjuntos de ocurrencias concretas de cada recurso. Por tanto, en lugar de decir, p. ej., que tal recurso lingüístico es un anglicismo de frecuencia, o que se comporta como uno, será correcto decir que es un recurso asociado a un anglicismo de frecuencia o algo comparable.

Lo recién apuntado podrá parecer una verdad de Perogrullo, mas hacerlo explícito se justifica en la medida en que existen estudiosos que incurren en la imprecisión señalada. Por otra parte, aun si se trata meramente de una imprecisión expresiva, de una metonimia, es una metonimia confusa y más que fácil de evitar, lo cual basta para tacharla de inaceptable en el lenguaje científico. Además, tal imprecisión puede incluso llegar a contribuir, especialmente en el aula, a la estigmatización, por completo injustificada, de recursos lingüísticos perfectamente castizos; así, p. ej., si un estudiante de traducción inglés-español escucha una y otra vez a sus profesores decir que las construcciones pasivas con ser + participio constituyen anglicismos, es probable que termine por generar un rechazo genérico frente a dichas construcciones y que intente eliminarlas de su acervo lingüístico (vid. Echeverría 2011), lo que sería en realidad un despropósito, pues se trata de construcciones utilizadas hasta por el mismísimo Cervantes.

\section{CONCLUSIÓN}

En estas páginas esperamos haber contribuido a la comprensión de la interferencia lingüística de frecuencia y de algunos de los problemas relacionados con dicho fenómeno. Lo aquí expuesto sobre el tema se puede sintetizar en los puntos siguientes:

1. La interferencia de frecuencia consiste en el empleo de un recurso existente como realización efectiva en la lengua pertinente con una frecuencia anormal, producto del conocimiento que tiene el sujeto hablante de otra lengua. Se trata, por tanto, de una desviación interferencial cuantitativa, que ocurre no respecto del sistema de la lengua, sino solo respecto de su norma. 
2. Tal desviación puede ser interlingüísticamente convergente o divergente; es decir, puede consistir en el uso preferencial de recursos que cuenten con un recurso correspondiente (idéntico o análogo) en la lengua desde la que se produce la interferencia o bien en el uso preferencial de recursos que carezcan de tal correspondencia.

3. Todo fenómeno de interferencia de frecuencia tiene una cara positiva, consistente en el empleo preferencial de ciertos recursos lingüísticos, y una cara negativa, consistente en el uso minoritario o nulo de otros recursos. En este sentido, este tipo de interferencia no se diferencia de los demás.

4. Ya que la interferencia de frecuencia no implica necesariamente problemas comunicativos, a pesar de relacionarse con tales problemas de manera ocasional, y ya que incluso habrá oportunidades en que, además de no causar problema comunicativo alguno, se presentará como una realidad imperceptible en inmediatez del discurso, aun para destinatarios leídos y reflexivos, no existe ninguna razón para censurar de modo genérico dicho fenómeno en la formación lingüística de bilingües.

5. Más injustificado aún que censurar de modo genérico la interferencia de frecuencia es censurarla solo en su forma de interferencia de convergencia, ignorando los casos de divergencia, ya que ambos fenómenos, desde el punto de vista de su resultado con respecto a la lengua pertinente, son en realidad análogos.

6. En cuanto a los términos como anglicismo de frecuencia, estos han de seguir reservándose para designar exclusivamente casos de interferencia de convergencia, ya que solo en estos se constata la imitación de otro idioma, mientras que en los casos de interferencia de divergencia sucede lo opuesto.

7. El usar tales términos para designar, en los casos de interferencia de convergencia, los recursos sobreutilizados en sí mismos, y no solo el sobreuso como tal, constituye una imprecisión que ha de evitarse. Conviene, pues, distinguir en todo momento entre, por un lado, anglicismos de frecuencia, catalanismos de frecuencia, galicismos de frecuencia, etc., y, por otro lado, los recursos lingüísticos asociados a ellos. 


\section{REFERENCIAS BIBLIOGRÁFICAS}

Alcaraz Varó, EnriQue y María Antonia Martínez Linares. 1997. Diccionario de lingüística moderna. Barcelona: Ariel.

Baetens Beardsmore, Hugo. 1986. Bilingualism: Basic Principles. Clevendon: Multilingual Matters.

Barkman, L. Bruce. 1968. Bilingualism and the Teacher of English as a Foreign Language. En Monika Kehoe (ed.). Applied Linguistics: A Survey for Language Teachers, pp. 58-72. Nueva York: Collier-Macmillan International.

Blas Arroyo, José Luis. 1991. Problemas teóricos en el estudio de la interferencia lingüística. Revista española de lingüística 21 (2): 265-290.

Brown, H. Douglas. 2000. Principles of Language Teaching and Learning. White Plains: Longman.

Cardinal, Pierre. 2009. VocabulaIDE: Influences de l'anglais - vraies et pretendues - et usages en transition. Ottawa: Université d'Ottawa.

CoRder, S. P. 1967. The significance of learner's errors. International Review of Applied Linguistics 5 (4): 161-170.

Coseriu, Eugenio. 1977. Sprachliche lnterferenz bei Hochgebildeten. En Herbert Kolb y Hartmut Lauffer (eds.). Sprachliche Interferenz: Festschrift für Werner Betz, pp. 77-100. Tubinga: Max Niemeyer Verlag.

1978. Sincronía, diacronía e historia: el problema del cambio lingüistico. Madrid: Gredos.

1982a. Determinación y entorno: dos problemas de una lingüística del hablar. En Teoría del lenguaje y lingüistica general: cinco estudios, pp. 282-323. Madrid: Gredos.

1982b. Sistema, norma y habla. En Teoría del lenguaje y lingüistica general: cinco estudios, pp. 11-113. Madrid: Gredos.

Darbelnet, JEAN. 1976. Le français en contact avec l'anglais en Amérique du Nord. Québec: P de l'U Laval.

Debyser, Francis. 1970. La linguistique contrastive et les interférences. Langue française 8: 31-61.

Dubois, Jean, Mathée Giacomo, Louis Guespin, Christiane Marcellesi, Jean-Baptiste Marcellesi y Jean-Pierre Mével. 2002. Dictionnaire de linguistique. París: Larousse.

Echeverría Arriagada, Carlos I. 2011. Sobre el uso de adverbios en -mente en la traducción inglés-castellano. Ponencia presentada en el XI Congreso Nacional de Estudiantes de Traducción e Interpretación, Valparaíso, Chile.

2014a. La interferencia lingüística de frecuencia: caracterización del fenómeno y reflexiones relacionadas a la enseñanza de la traducción. Ponencia presentada en las III Jornadas Nacionales de la Enseñanza de la Traducción, Concepción, Chile.

2014b. La interferencia lingüística de frecuencia y el uso de términos como "anglicismo de frecuencia". Ponencia presentada en las VI Jornadas de Investigación de la Facultad de Humanidades de la Universidad de Santiago de Chile, Santiago, Chile.

2015. Actitudes y preferencias de estudiantes de traducción inglés-español frente a recursos gramaticales del español asociados a anglicismos de frecuencia. Tesis de Magíster, Universidad de Santiago de Chile.

EluIs, Rod. 1994. The Study of Second Language Acquisition. Oxford: Oxford University.

Gass, Susan M., Jennifer Behney y Luke Plonsky. 2013. Second Language Acquisition: An Introductory Course. Nueva York: Routledge. 
Gold, DAvid L. 1983. Frequency Gallicism, Frequency Anglicism. Language Problems \& Language Planning 7 (3): 360-361.

Gómez CAPUZ, JuAn. 1998. El préstamo lingüistico: conceptos, problemas y métodos. Valencia: Universitàt de Valencia.

Granda, Germán DE. 1968. Transculturación e interferencia lingüistica en el Puerto Rico contemporáneo (1898-1968). Bogotá: Instituto Caro y Cuervo.

Haugen, Einar. 1956. Bilingualism in the Americas: A Bibliography and Research Guide. Alabama: University of Alabama.

HeRnÁNDEZ GarCía, CARMEN. 1998. Una propuesta de clasificación de la interferencia lingüística a partir de dos lenguas en contacto: el catalán y el español. Hesperia: anuario de filología hispánica 1: 61-80.

Huelmslev, Louis. 1971. Prolegómenos a una teoría del lenguaje. Madrid: Gredos.

Kabatek, Johannes. 1997a. Dime cómo hablas y te diré quién eres. Mezcla de lenguas y posicionamiento social. Revista de antropología social 6: 215-236.

1997b. Zur Typologie sprachlicher Interferenzen. En Wolfgang W. Moelleken y Peter J. Weber (eds.). Neue Forschungsarbeiten zur Kontaktlinguistik, pp. 232-241. Bonn: Dümmler.

2000. Os falantes como lingüistas. Tradición, innovación e interferencias no galego actual. Vigo: Xerais.

LAdO, RoBert. 1973. Lingüistica contrastiva. Lenguas y culturas. Madrid: Alcalá.

López Morales, Humberto. 1993. Sociolingüística. Madrid: Gredos.

Lorenzo, Emilio. 1980. El anglicismo en la España de hoy. El español de hoy, lengua en ebullición, pp. 96-121). Madrid: Gredos.

1996. Anglicismos hispánicos. Madrid: Gredos.

Mackey, William F. 1970. Interference, Integration and the Synchronic Fallacy. En James E. Alatis (ed.). Georgetown University Round Table on Languages and Linguistics 1970: Bilingualism and Language Contact, pp. 195-227. Washington: Georgetown University.

Martínez de Sousa, José. 2008. Diccionario de usos y dudas del español actual. Gijón: Trea.

Mauranen, Anna. 2009. Introduction. En Anna Mauranen y Elina Ranta (eds.). English as a Lingua Franca: Studies and Findings, pp.1-7. Cambridge: Cambridge Scholars.

McLaughlin, Barry. 1984. Second-Language Acquisition in Childhood. Nueva Jersey: Lawrence Erlbaum Associates.

Odun, T. 1989. Language Transfer: Cross-Linguistic Influence in Language Learning. Cambridge: Cambridge University.

Overbecke, Maurits van. 1976. Mécanismes de l'interférence linguistique. Madrid: Fragua.

PisAni, VitToRe. 1947. La lengua e la sua storia. En Lingüistica generale e indoeuropea, pp. 9-19. Milán: Librería Editrice Scientifico-Universitaria.

Pountain, Christopher J. 1994. Syntactic anglicisms in Spanish: innovation or exploitation? En M. Mair Parry, Winifred V. Davies y Rosalind A. M. Temple (eds.). The Changing Voices of Europe: Social and Political Changes and their Linguistic Repercussions, Past, Present and Future, pp. 109-124. Cardiff: University of Wales.

Real Academia Española. 2014. Diccionario de la lengua española. Madrid: Espasa Libros.

Rodríguez Medina, María Jesús. 2000. Los anglicismos de frecuencia sintácticos en los manuales de informática traducidos. Tesis doctoral, Universidad de Las Palmas de Gran Canaria [en línea]. Disponible en http://acceda.ulpgc.es/handle/10553/2100?nitem=0\&a nterior $=\&$ siguiente $=[$ consulta $04 / 12 / 2014]$.

2002. Los anglicismos de frecuencia sintácticos en español. Revista española de lingüística aplicada 15: 149-170.

SAussure, Ferdinand DE. 2005. Curso de lingüistica general. Buenos Aires: Losada. 
Thomason, Sarah Grey. 2001. Language Contact: An Introduction. Bodmin, Cornwall: Edinburgh University.

Thomason, Sarah Grey y Terrence Kaufman. 1988. Language Contact, Creolization, and Genetic Linguistics. Berkeley: University of California.

VÁzQUeZ-Ayora, GeRARDo. 1977. Introducción a la traductología: curso básico de traducción. Washington: Georgetown University.

Véliz Ojeda, Eduardo y Víctor Cámara Cámara. 2010. La "parataxis" como anglicismo de frecuencia en traducciones del inglés al castellano: análisis desde los estudios culturales. Ponencia presentada en el X Congreso de Estudiantes de Traducción e Interpretación, Copiapó, Chile.

Viaggio, Sergio. 2004. Teoría general de la mediación interlingüe. Alicante: Universidad de Alicante.

WeinReich, Uriel. 1974. Lenguas en contacto: descubrimientos y problemas. Caracas: Universidad Central de Venezuela. 
